\title{
SY66-1
}

\section{Calcium permeable ion channels and cell death pathways in breast cancer cells}

\section{Gregory R. Monteith}

\author{
School of Pharmacy, The University of Queensland, Australia
}

The calcium signal has a vital role in many processes important in breast cancer progression, including cellular proliferation and migration. An increasing number of calcium permeable ion channels including TRPV6 and TRPV4 have been linked to breast cancers of the basal molecular subtype, which have a significant overlap with triple negative breast cancers; a subtype where the need for new therapeutic approaches has been highlighted. Pharmacological inhibition of calcium permeable ion channels which are a feature of breast cancers of the basal molecular subtype represent new opportunities to attenuate breast cancer cell proliferation and/or migration. Calcium signalling also plays a critical role in the initiation or modulation of cell death pathways, however, the possible opportunity of targeting calcium permeable ion channels in triple negative breast cancer cells has been less extensively investigated. Our studies demonstrated that pharmacological activation of TRPV4 in MDA-MB-468 triple negative basal-like breast cancer cells can induce oncosis and apoptosis. Genetically encoded calcium indicators when combined with automated epifluorescence microscopy is now providing new insights into calcium signalling in triple negative breast cancer cells during apoptotic and necrotic stimuli. 